\title{
Construction of expression vectors of capsid proteins from goose parvovirus and investigation of the immunogenicity
}

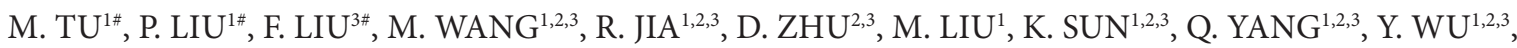 \\ X. CHEN ${ }^{2,3}$, A. CHENG ${ }^{1,2,3^{*}}$, S. CHEN ${ }^{1,2,33^{*}}$
}

\begin{abstract}
${ }^{1}$ Institute of Preventive Veterinary Medicine, Sichuan Agricultural University, Wenjiang District, Chengdu City, Sichuan Province, 611130, P. R. China; ${ }^{2}$ Avian Disease Research Center, College of Veterinary Medicine of Sichuan Agricultural University, Wenjiang District, Chengdu City, Sichuan Province, 611130, P. R. China; ${ }^{3}$ Key Laboratory of Animal Disease and Human Health of Sichuan Province, Sichuan Agricultural University, Wenjiang District, Chengdu City, Sichuan Province, 611130, P. R. China
\end{abstract}

\section{Received October 2, 2017; revised December 7, 2017; accepted October 8, 2018}

\begin{abstract}
Summary. - Goose parvovirus (GPV) is a highly contagious and lethal disease in goslings and Muscovy ducklings, and is of concern to the waterfowl industry. With the aim of comparing the cellular immunogenicity of three capsid proteins of GPV, plasmids of pcDNA3.1(+)-VP1, pcDNA3.1(+)-VP2, and pcDNA3.1(+)-VP3 were constructed, and the recombinant protein VPs were expressed using an eukaryotic expression system. We detected the levels of immune-related genes (CD4, CD8 $\alpha$, IL-1 $\beta$, IL-6, IFN $\alpha$, IFN $\gamma$, and IFN $\lambda$ ) in both goose embryo fibroblasts (GEF) and goose peripheral blood mononuclear cells (PBMCs) cellular models. The immune response conferred by a VP2 DNA vaccine in vivo was observed in a time course. Our data suggested that the cellular immune response to VP2 and VP3 was stronger than that to VP1, while VP2 and VP3 shared similar cellular immune reactivity. In addition, vaccination with VP2 plasmid can induce high level of IgY antibody that continued to increase through 28 days post vaccination. Therefore, our findings shed light on the host cellular immune response against GPV capsid proteins.
\end{abstract}

Keywords: GPV; capsid proteins; cellular immune response; humoral immunity

\section{Introduction}

Derzsy's disease is an acute infectious disease caused by GPV that can cause low spirits, loss of feathers, stunted growth, diarrhea and neurological symptoms in geese (Palya et al., 2009). Infected geese have typical conditions such as hydropericardium, ascites, enteritis, and degenerative and inflammatory changes of the myocardium and liver (Jansson et al., 2007). GPV is a small icosahedral virus with a

*Corresponding authors. E-mails: shunchen@sicau.edu.cn (Shun Chen); chenganchun@vip.163.com (Anchun Cheng); phone: +86-028-86291482. "These authors contributed equally as co-first authors of this work.

Abbreviations: GEF = goose embryo fibroblasts; GPV = goose parvovirus; $\mathrm{HRP}=$ horseradish peroxidase; $\mathrm{PBMCs}=$ peripheral blood mononuclear cells; PBS = phosphate buffered saline; RTqPCR = real-time quantitative PCR; VLPs = virus-like particles diameter of 20-24 nm, that belongs to the Dependovirus genus of the Parvoviridae family (Cotmore et al., 2014). It has an approximately $5-\mathrm{kb}$ single genome that contains two open reading frames (ORF). The left ORF encodes a nonstructural protein that is responsible for viral replication, and the right ORF encodes three capsid proteins, VP1, VP2, and VP3 (Gall-Reculé and Jestin, 1994). VP1 contains the entire sequence of VP2 and VP3, all three VPs share the same terminal codon. The VP1 N-terminal region harbors a nuclear localization signal and a PLA domain, which is critical for viral infectivity (Lombardo et al., 2002; Vihinenranta et al., 2002; Ros et al., 2006). VP2 has an "anti-receptor" that can interact with a cellular receptor on the cell surface (Chipman et al., 1996; Summerford et al., 1998). VP3 appears to be the most abundant of the three capsid proteins and may act as a scaffold protein (Ju et al., 2011).

China is the world's largest waterfowl breeder. The regulations for producing poultry meat and eggs are increasing, 
and the living standards are improving. GPV is of concern to the poultry industry because of its rapid transmission and high mortality rates in infected animals. At present, there are no effective drugs for GPV treatment, and preventative measures involve inoculation of traditional vaccines or hyperimmune serum in breeding geese. GPV poses a significant threat to the goose industry, and insight into its pathogenic mechanisms and molecular biology characteristics is needed. The humoral immunity responses of VPs have been widely discussed (Jaiwei et al., 2010, 2013; Chen et al., 2012; Xu et al., 2014). However, there is still a lack of information on the cellular immunogenicity of VPs.

In this study, we investigated the differences in cellular immune responses of the three GPV capsid proteins. Recombinant VPs were expressed in an eukaryotic expression system and used as antigens in the cellular models to determine which ones have the best reactivity. VP2 was selected as an immunogen and further characterized by examining its effects on expression of various cytokine genes in goose peripheral blood cells as well as in GEF and by determining the titer of antigen-specific antibodies in serum. Our data showed that the cellular immunity against VP2 and VP3 was stronger than that against VP1 and that the pcDNA3.1(+)VP2 was able to induce GPV-specific humoral response in immunized goslings. This work provides essential data for the development of GPV vaccines.

\section{Materials and Methods}

Plasmid construction. To generate the plasmids pcDNA3.1(+)VP1, pcDNA3.1(+)-VP2, and pcDNA3.1(+)-VP3, DNA fragments were amplified from the GPV genome (GenBank Acc. No. U25749) by PCR using specific primers (Table 1). The length of PCR products of VP1, VP2 and VP3 are 2216, 1785 and 1625 bp, respectively. The products were digested with the restriction enzymes KpnI and NotI and cloned into the corresponding sites of pcDNA3.1 (+) (Invitrogen, Germany). Additionally, the His-tag sequences were inserted into the reverse primer. The plasmids were transformed into Escherichia coli $\mathrm{DH} 5$ a and purified using the EndoFree plasmid purification Kit (OMEGA, USA).
Cell culture, transfection and the expression of VPs. GEF were grown in modified Eagle's medium supplemented with $10 \%$ FBS. GEF were transfected with $2.5 \mu \mathrm{g} /$ well pcDNA3.1(+)-VP1, pcDNA3.1(+)-VP2, or pcDNA3.1(+)-VP3 using Lipofectmi$\mathrm{ne}^{\mathrm{TM}} 3000$ (Invitrogen, USA) according to the manufacturer's instructions and incubated for $48 \mathrm{~h}$. Cells were lysed with a lysis buffer containing $25 \mathrm{mmol} / \mathrm{l}$ Tris-HCl, pH 7.6, $150 \mathrm{mnol} / \mathrm{l} \mathrm{NaCl}, 1 \%$ NP- $40,1 \%$ sodium deoxycholate, and $0.1 \%$ SDS for $20-30$ min on ice, and whole cell lysate was obtained by subsequent centrifugation. Lysates were boiled for $10 \mathrm{~min}$, separated on $12 \%$ polyacrylamide slab gels and transferred to polyvinylidene fluoride membrane (biorad, USA) for Western blot analysis. The blots were incubated with an anti-His monoclonal antibody (1:6,000, Protein, USA) or antiGPV antiserum (1:1,000), followed by incubation with horseradish peroxidase (HRP)-conjugated goat anti-mouse antibody $(1: 10,000$, EARTH, USA). The immune complex was detected by Pierce ${ }^{\mathrm{TM}} \mathrm{ECL}$ western blotting substrate (Thermo, MA, USA).

Goose PBMC infection model. Blood from healthy adult geese was collected in an anticoagulant-containing vial. PBMCs were isolated using the Goose Lymphocyte Separation Medium kit (TBD sciences, Tianjing, China) and grown in RPMI-1640 with $10 \%$ serum at a concentration of $1 \times 10^{7}$ cells $/ \mathrm{ml}$. Briefly, blood was overlaid on an equal volume of RPMI-1640, and then the mixture was slowly added into lymphocyte separation medium (2:1) with centrifugation at $800 \times \mathrm{g}$ for $30 \mathrm{~min}$. The interface containing the PBMCs was collected and washed twice in wash buffer and then resuspended in RPMI-1640 medium. Forty-eight hours after the GEF were transfected with pcDNA3.1(+)-VP1, pcDNA3.1(+)-VP2, pcDNA3.1(+)-VP3, or negative control pcDNA3.1(+) vector, the cells were harvested and lysed by three freeze/thaw cycles, and then the supernatants were collected. PBMCs incubated with the supernatant from the transfected cells for $6 \mathrm{~h}$ were examined for differences in expression of immune-related genes (CD4, CD8 $\alpha$, IL-6, IL- $1 \beta$, and IFNs) by real-time quantitative PCR (RT-qPCR).

GEF infection model. GEFs were transfected with pcDNA3.1(+)VP1, pcDNA3.1(+)-VP2, pcDNA3.1(+)-VP3, and pcDNA3.1(+) for $48 \mathrm{~h}$. The transfected cells were washed with cold phosphate buffered saline (PBS) three times and then resuspended in RNAiso Plus regent (Takara Bio, Otsu, Japan). RT-qPCR was used to detect the transcripts of goose immune-related genes (CD4, CD8 $\alpha$, IL-6, IL- $1 \beta$, and IFNs).

Table 1. The list of primers for plasmid construction

\begin{tabular}{llr}
\hline Genes & The sequences (5'-3') & The site \\
\hline VP1 & F: ACTTAAGCTTGGTACCGCCACCATGTCTACTTTTTTAGATTC & $K p n \mid$ \\
& R: CTAGACTCGAGCGGCCGCTTAGTGGTGGTGGTGGTGGTGCAGATTTTGAGTTAGATATC & Not $\mid$ \\
VP2 & F: ACTTAAGCTTGGTACCGCCACCATGGCACCTGCAAA & $K p n \mid$ \\
& R: CTAGACTCGAGCGGCCGCTTAGTGGTGGTGGTGGTGGTGCAGATTTTGAGTTAGATATC & Not $\mid$ \\
VP3 & F: ACTTAAGCTTGGTACCGCCACCATGGCAGAGGGAGGAG & $K p n \mid$ \\
& R: CTAGACTCGAGCGGCCGCTTAGTGGTGGTGGTGGTGGTGCAGATTTTGAGTTAGATATC & Not $\mid$ \\
\hline
\end{tabular}


Table 2. The list of primers for RT-qPCR

\begin{tabular}{|c|c|c|c|}
\hline Target gene & Primer sequences $\left(5^{\prime}-3^{\prime}\right)$ & Products & Reference \\
\hline GAPDH-F & CATTTTCCAGGAGCGTGACC & $80 \mathrm{bp}$ & \\
\hline GAPDH-R & AGACACCAGTAGACTCCACA & & \\
\hline IL-1 $\beta-F$ & TCCGCCAGCCGCAAAGTG & 136 bp & (Qi et al., 2015) \\
\hline IL- $1 \beta-\mathrm{R}$ & CGCTCATCACGCAGGACA & & \\
\hline IL-6-F & AAGTTGAGTCGCTGTGCT & $120 \mathrm{bp}$ & (Qi et al., 2015) \\
\hline IL-6-R & GCTTTGTGAGGAGGGATT & & \\
\hline CD4-F & TTTCAACGCCACAGCAGA & $127 \mathrm{bp}$ & (Chen et al., 2015) \\
\hline CD4-R & GTGCCTCAACTGGATTTT & & \\
\hline $\mathrm{CD} 8 \alpha-\mathrm{F}$ & AGAGACGAGCAAGGAGAA & $97 \mathrm{bp}$ & (Chen et al., 2015) \\
\hline CD8a-R & GACCAGGGCAATGAGAAG & & \\
\hline IFNa-F & CAGCACCACATCCACCAC & $98 \mathrm{bp}$ & (Chen et al., 2015) \\
\hline IFNa-R & TACTTGTTGATGCCGAGGT & & \\
\hline IFN $\gamma-\mathrm{F}$ & TGAGCCAGATTGTTTCCC & 146 bp & (Chen et al., 2015) \\
\hline IFN $\gamma-\mathrm{R}$ & CAGGTCCACGAGGTCTTT & & \\
\hline IFN $\lambda-F$ & GAGCTCTCGGTGCCCGACC & $165 \mathrm{bp}$ & \\
\hline IFN $\lambda-R$ & CTCAGCGGCCACGCAGCCT & & \\
\hline
\end{tabular}

Analysis of immune response genes by RT-qPCR. RNA extraction and cDNA synthesis: The cells were centrifuged for $5 \mathrm{~min}$, and the resulting pellet was resuspended in $1 \mathrm{ml}$ of RNAiso Plus regent (Takara Bio, Otsu, Japan); total RNA was isolated. Briefly, RNA from the resulting mixture was extracted with chloroform and precipitated with isopropanol. The RNA pellet was washed with 75\% ethanol and resuspended in RNA-free water (TIAGEN, Beijing, China). cDNA was synthesized using a 5x All-In-One RT Master Mix Reagent Kit according to the manufacturer's instructions (Applied Biological Materials, Richmond, BC, Canada).

RT-qPCR: The mRNA expression levels of goose CD4, CD8 $a$, IL-6, IL- $1 \beta$, and IFNs were analyzed by RT-qPCR using the QuantiTect SYBR Green qPCR Kit (Qiagen, CA, USA) on a CFX96 real time system (Bio-Rad, CA, USA) using published gene-specific primers (Table 2). GAPDH was used as the reference gene. QPCR was performed in triplicate for each sample in a total volume of $10 \mu \mathrm{l}$ consisting of $5 \mu \mathrm{l}$ of QuantiTect SYBR Green Master Mix (Qiagen, CA, USA $), 0.2 \mu \mathrm{l}$ cDNA, and $0.2 \mu \mathrm{l}(5 \mu \mathrm{mol} / \mathrm{l})$ of each primer. Products were amplified during the following program: 1 cycle at $94^{\circ} \mathrm{C}$ for $3 \mathrm{~min}$, followed by 39 cycles of $94^{\circ} \mathrm{C}$ for $10 \mathrm{~s}$, and 1 cycle at $60^{\circ} \mathrm{C}$ for $30 \mathrm{~s}$. The final step was to obtain a melt curve for the PCR products to determine the specificity of the amplicons. Expression levels were calculated relative to the GAPDH expression.

Immunization of goslings with plasmids. Goslings ( 9 per experimental group) were immunized with $200 \mu \mathrm{g}$ of plasmid dissolved in $100 \mu$ of sterile PBS by i.m. route twice, with two weeks between inoculations. As negative and untreated controls, the goslings were injected with empty pcDNA3.1 (+) vector or PBS alone, respectively. At $0,7,14,21$, and 28 days post immunization, blood samples were collected from the jugular vein, and one portion was mixed with RNAiso Plus reagent (Takara Bio, Otsu, Japan) for analysis of immune-related genes expression (CD4, CD8 $\alpha$, IL-6, IL-1 $\beta$, and IFNs) by RT-qPCR. A second portion of the collected serum samples were stored at $-20^{\circ} \mathrm{C}$ until they could be analyzed for antibodies against GPV by ELISA.

The concentration of serum IgY antibodies against GPV was determined by ELISA. Briefly, individual wells were coated with $30 \mathrm{ng} /$ well of the purified GPV and blocked with $1 \%$ gelatin. The coated GPV antigens were incubated with serum samples diluted at $1: 80$ at $37^{\circ} \mathrm{C}$ for $1.5 \mathrm{~h}$. After washing, the wells were incubated with HRP-conjugated goat anti-Bird IgY (abcam, USA) diluted 1:3,500. Bound antibodies were detected following the addition of $100 \mu \mathrm{l} /$ well of 3, 3', 5, 5'-tetramethylbenzidine. The absorbances at $450 \mathrm{~nm}$ and $630 \mathrm{~nm}$ were read using a microplate reader.

Statistical analysis. Data are shown as the mean and standard deviation calculated using Prism 5 (GraphPad, USA), with Student's $t$-test analysis of variance.

\section{Results}

\section{Expression of VP1, VP2, and VP3 in GEF}

Forty-eight hours after transfection with pcDNA3.1(+)VP plasmids, VP1, VP2, and VP3 recombinant proteins in the cell lysates of transfected GEF were analyzed by Western blotting. All three VPs were recognized by specific anti-His antibody and anti-GPV antiserum at approximately $80 \mathrm{kDa}$, $70 \mathrm{kDa}$ and $60 \mathrm{kDa}$, respectively (Fig. 1).

VPs-induced cellular immune response in different cellular models

The immunogenicity of capsid proteins was examined in different cellular models. In the GEF model, cells were 
transfected with pcDNA3.1(+)-VP1, pcDNA3.1(+)-VP2, pcDNA3.1(+)-VP3, and pcDNA3.1(+) for $48 \mathrm{~h}$, and RNA was extracted using a RNAiso Plus reagent (Takara Bio, Otsu, Japan). The transcripts of the genes encoding CD4, CD8 $\alpha$, IL-6, IL-1 $\beta$, and IFNs were measured. The results indicated that VP2 and VP3 significantly up-regulated the transcription of genes encoding IL- 6 and IL- $1 \beta$ compared with the vector control $(\mathrm{P}<0.001)$, while IL-6 was significantly increased by VP2 when compared with VP3 and the vector control $(\mathrm{P}<0.01)$ (Fig. 2). The transcripts of the genes encoding CD4, CD8 $\alpha, \operatorname{IFN} \alpha, \operatorname{IFN} \gamma, \operatorname{IFN} \lambda$ were nearly undetectable (data not shown) in both transfected and control cells.

In the PBMC model, none of the genes were altered by the treatment with VP1-containing extracts compared to the treatment with extracts from the control group. The transcripts of the genes encoding CD8a and IL- $1 \beta$ were significantly increased by the treatment with VP3-containing lysates compared with VP1 extracts. The transcript levels for IL-6, IL-1 $\beta$, IFN $\gamma$, and IFN $\lambda$ were significantly upregulated by VP2 extracts when compared to control and VP1 extracts (Fig. 3).

Expression levels of cytokines in the peripheral blood cells from vaccinated goslings

Based on the in vitro data, pcDNA3.1(+)-VP2 was used as a DNA vaccine to immunize goslings. Blood samples were collected at day $7,14,21$, and 28 post-vaccination for cytokine expression analysis. Real time qPCR was performed to measure the mRNA expression levels of goose CD4, CD8 $\alpha$, IL-1 $\beta$, IL-6, IFN $\alpha$, IFN $\gamma$, and IFN $\lambda$. The results indicated that the transcriptional levels of the genes encoding CD4 and CD8 showed a steady increase through day 28 post immunization. At day 7 and day 14, the mRNA expression level of CD8 is up-regulated significantly compared to control

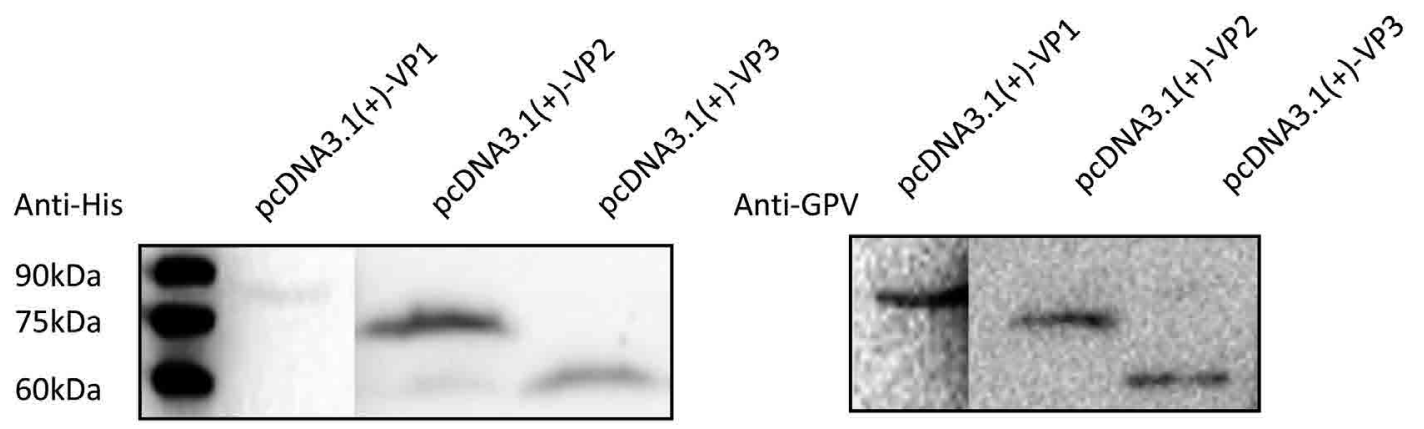

Fig. 1

Western blot analyses of VP1, VP2, and VP3 expression

Protein extracts from GEF transfected with the indicated plasmids were separated by gel electrophoresis and then transferred to PVDF membrane. The blot was incubated with anti-His antibody or anti-GPV antiserum, followed by a HRP-conjugated secondary antibody.

IL-1 $\beta$

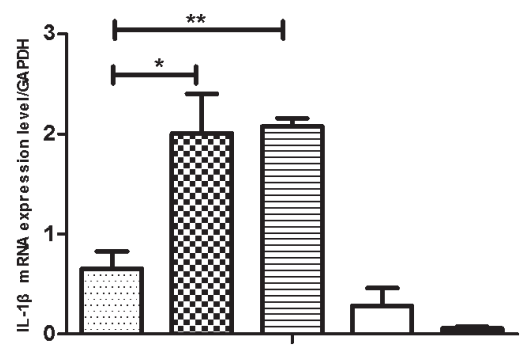

IL-6

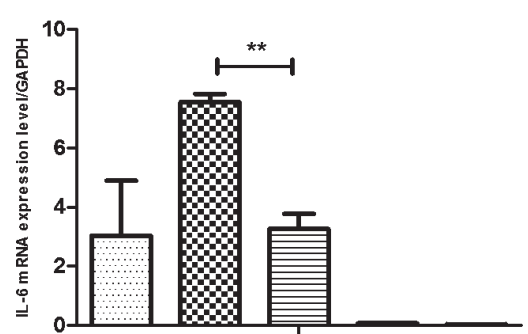

Fig. 2

mRNA expression levels of proinflammatory cytokines in goose embryo fibroblast after transfection with pcDNA3.1(+)-VPs and pcDNA3.1(+) Gene expression relative to the housekeeping gene GAPDH was assessed using qPCR. The data are expressed as the mean \pm SEM $(n=4)$, and the difference between groups was analyzed with a t-test. Groups denoted by $\left(^{*}\right)$ indicate a significant difference at $P<0.05$, and groups denoted by $\left({ }^{* *}\right)$ indicate a significant difference at $P<0.01$. 

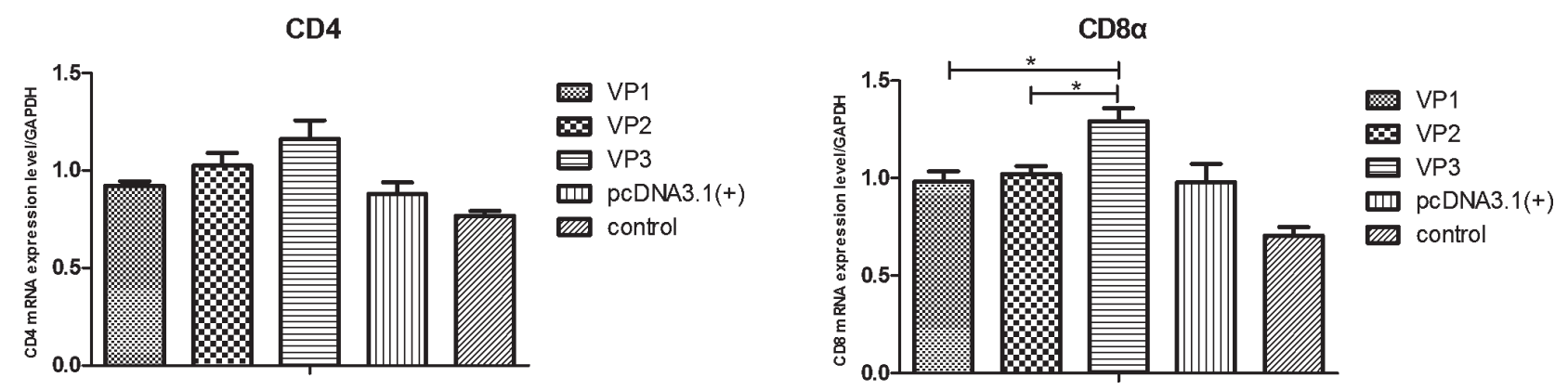

IL-1 $\beta$
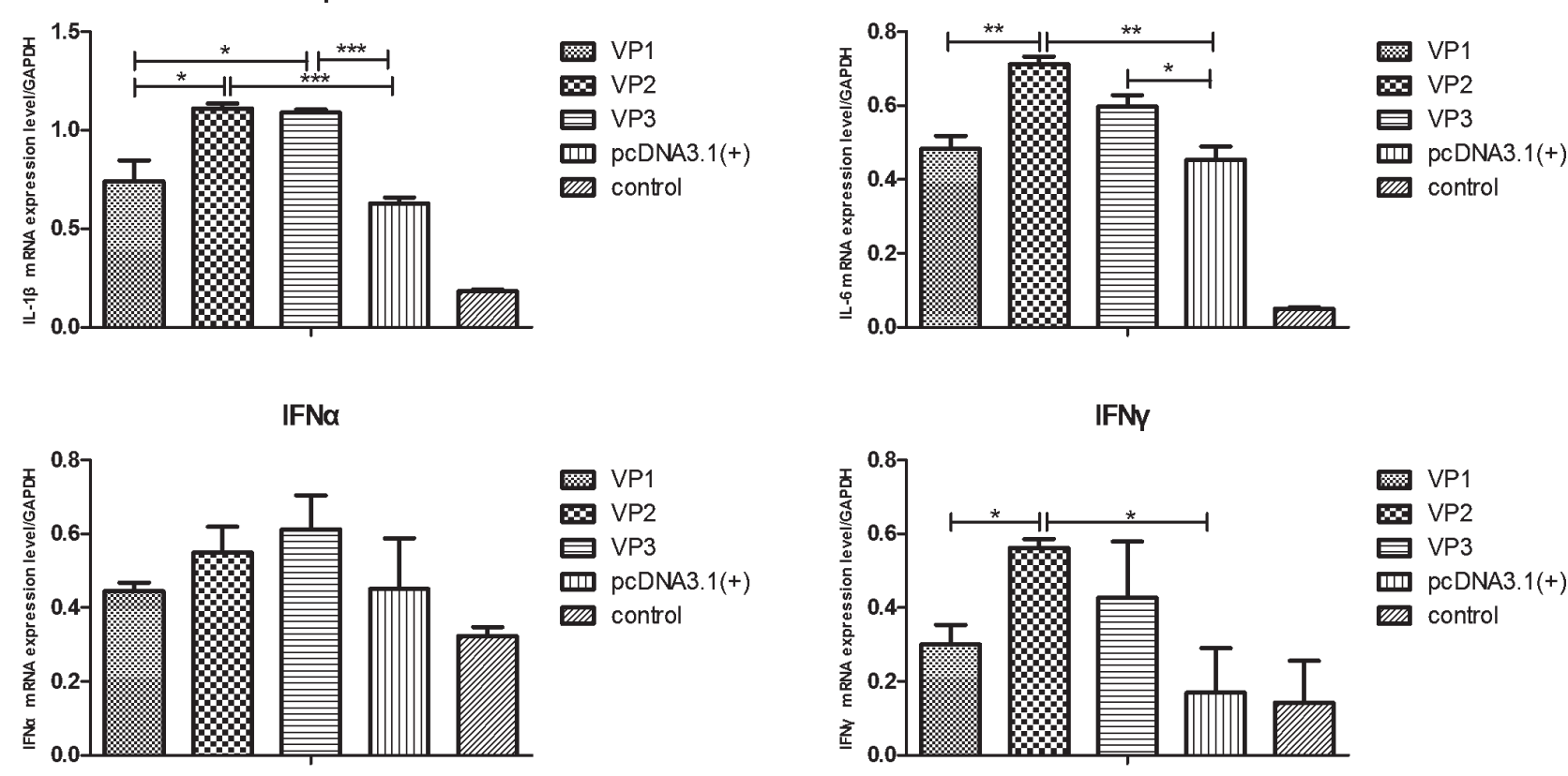

IFNA

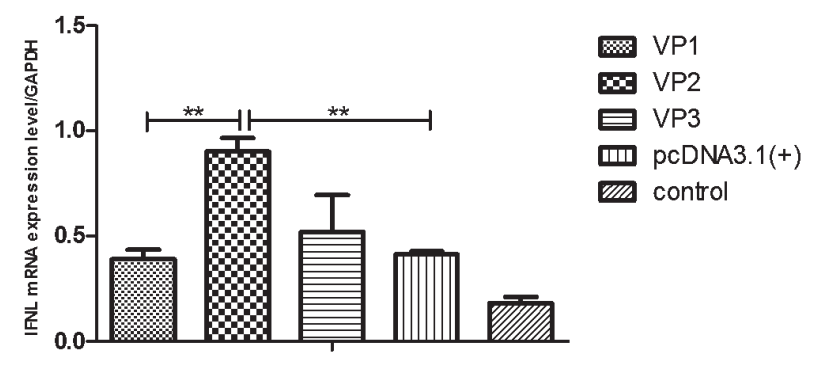

Fig. 3

mRNA expression levels of $T$ cell markers, proinflammatory cytokines and interferons after stimulation with VPs CD4, CD8 $\alpha$, IL-6, IL1- $\beta$, IFNa, IFN $\gamma$, and IFN $\lambda$ mRNA expressions were normalized using an internal control (GAPDH)

The data were indicated as the mean $\pm \operatorname{SEM}(\mathrm{n}=4)$. Groups denoted by $\left(^{*}\right)$ indicate a significant difference between experimental groups $\left({ }^{*}, P<0.05\right.$; $\left.{ }^{* *}, P<0.01 ;{ }^{* *}, P 0.001\right)$.

(Fig. 4). However, the mRNA expression levels of IL-1 $\beta$ and IFN $\gamma$ experienced a decline after 14 days. Additionally, the expression level of IL-18 significantly increased on day 21 . The data for IFNa and IFN $\lambda$ transcripts showed a similar trend in that they both peaked on day 14 and then declined to very low levels on day 21 through day 28 (Fig. 4).

\section{Detection of GPV-specific antibody in vaccinated goslings}

To determine the efficacy of vaccination with the VP2 DNA vaccine, ELISA was performed to measure specific antibodies. Our results demonstrated that at day 14 post-immunization, the GPV-specific antibody was highly abundant. In addition, the GPV-specific antibody in pcDNA3.1(+)-VP2 immunized goslings was significantly increased at day 21 and 


\section{CD4}

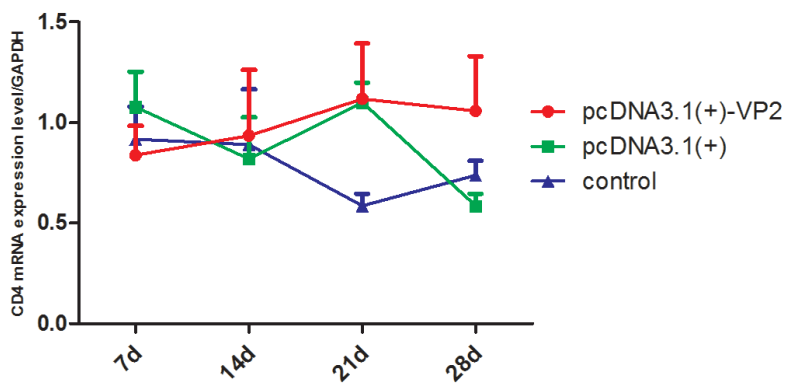

IL-1 $\beta$

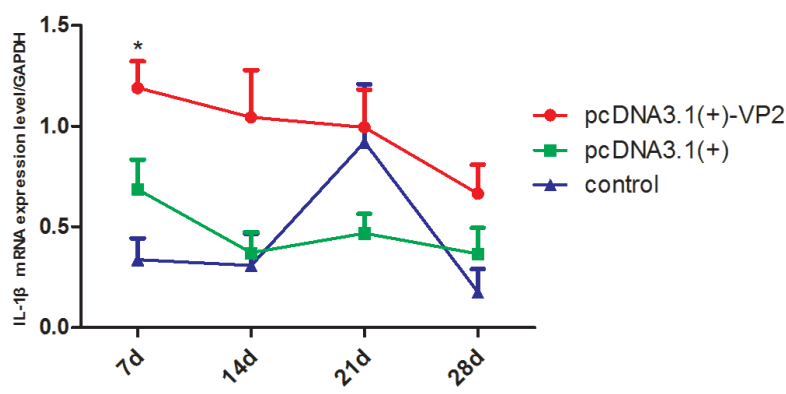

IFNY

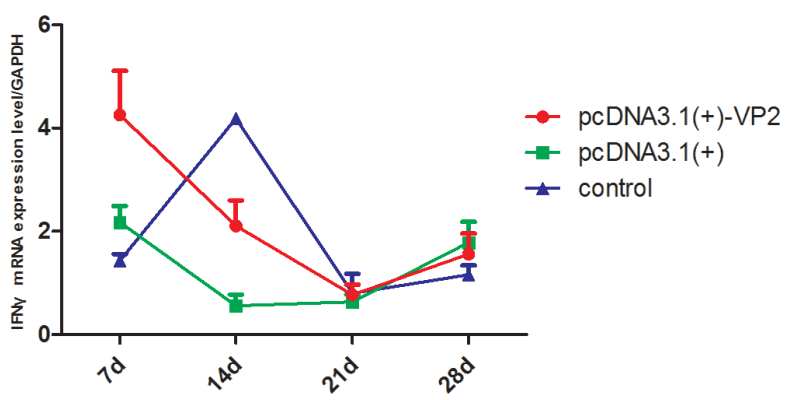

CD8 $\alpha$

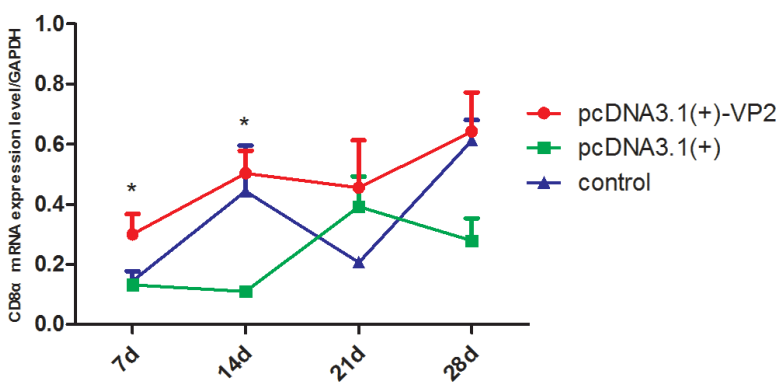

IFNa

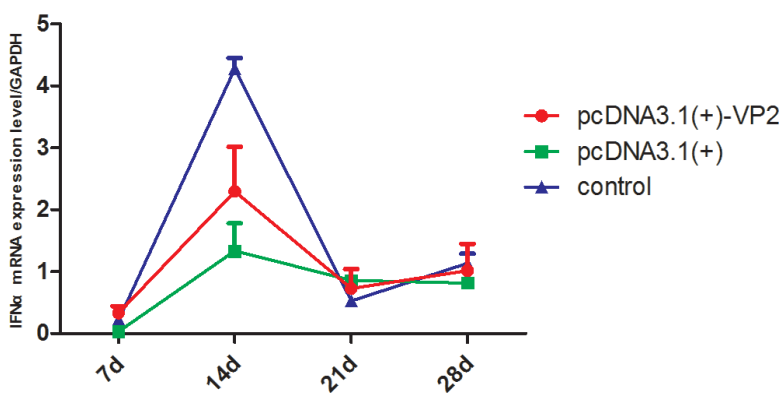

IFM

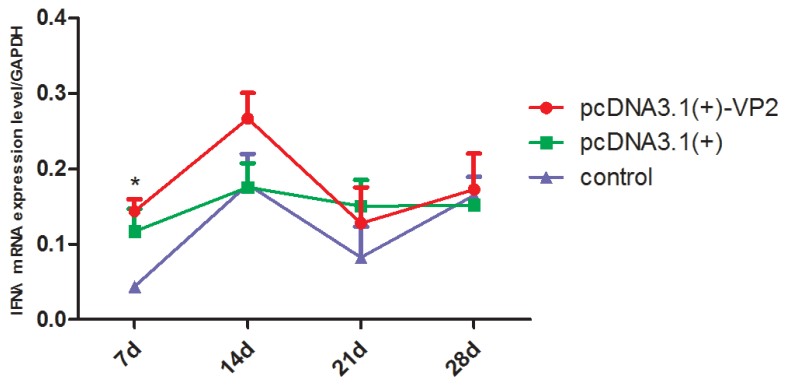

Fig. 4

pcDNA3.1(+)-VP2 plasmid induced different expression level of cytokines in goose peripheral blood cells At day 7, 14, 21, and 28 post-immunization, transcripts were detected by RT-qPCR, and GAPDH was used as an internal control. The significance between mRNA cytokine levels in goose peripheral blood cells was determined by a $t$-test.

day 28 compared to control groups, and the IgY antibody levels were higher at day 28 than at any other time during the experimental phase (Fig. 5).

\section{Discussion}

Here, we examined the immunogenicity of recombinant VPs in different cellular models by assaying the expression level of various immune-related genes and the antibody titers in order to gain insight into the molecular mechanisms involved in the protective responses conferred by a GPVspecific DNA vaccine. The hallmark cytokine of the Th1 response, IFN $\gamma$, plays a major role in both innate and adaptive immunity. The results of this study indicate that IFN $\gamma$ is significantly upregulated in PBMCs following treatment with VP2-containing extracts. Pro-inflammatory cytokines such as IL-1 $\beta$ play an important role in the host inflammatory and immune reactions. In assessing the transcriptional levels of genes encoding several cytokines, our results suggest that capsid proteins VP2 and VP3 significantly induced expression of IL- $1 \beta$ in GEF and goose PBMCs. A previous study 


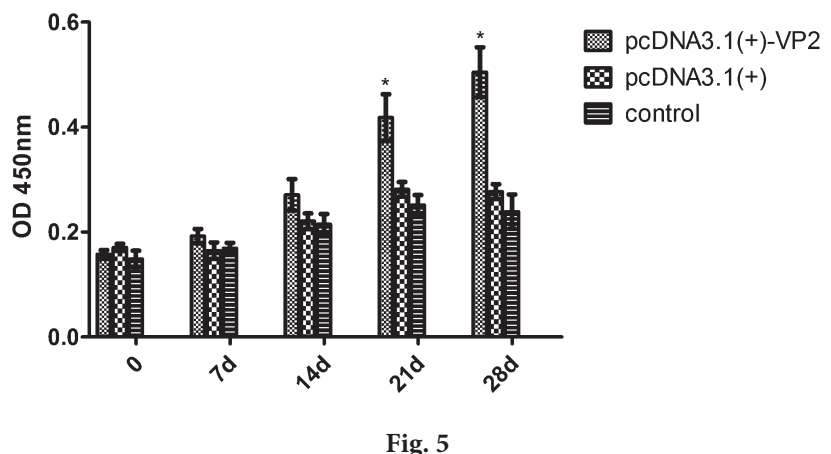

Detection of serum-specific IgY antibody against GPV by indirect ELISA

Goslings were immunized with pcDNA3.1(+)-VP2 or pcDNA3.1(+) vector as a control. Blood samples were obtained from individual goslings at the indicated time points post immunization, and the levels of serum IgY antibodies against GPV were determined by indirect ELISA. Data are indicated as the mean $\pm \operatorname{SEM}(n=4)$.

indicated that VP1, VP2, and VP3 from GPV expressed from a baculovirus expression vector in insect cells were detected and that the formation of virus-like particles (VLPs) was observed. It was also found that the recombinant proteins elicited an immunoreactive response with specific antibodies and that the neutralizing activities of the antibodies against VP2 and VP3 were higher than that of antibodies against VP1 (Ju et al., 2011). In this study, we found that both VP2 and VP3 can significantly upregulate the expression of genes encoding IL- 6 and IL- $1 \beta$ in the GEF model, while the expression levels of IL-6, IL- $1 \beta$, IFN $\gamma$, and IFN $\lambda$ were significantly upregulated in PBMCs following the treatment with VP2containing extracts. None of the tested genes showed any changes in response to VP1 in either the GEF or PBMCs. These results indicated that the cellular immune reactivity of VP2 and VP3 was stronger than that of VP1 and that VP2 is stronger than VP3. Since VP2 and VP3 are sub-fragments of VP1, VP1 theoretically contains all the antigenic epitopes on the VP2 and VP3 proteins. However, it is interesting that, based on our data, VP2 and VP3 appear to have higher immunogenicity than VP1. Among these three proteins, VP2 contains the immunodominat region the antibody can interact with. The VP2 proteins of many parvoviruses, including human parvovirus B19 (Lowin et al., 2010), canine parvovirus (Jin et al., 2012), porcine parvovirus (Zhou et al., 2010), and goose parvovirus (Chen et al., 2012) are able to form of VLPs in the baculovirus expression system. The VLPs always share similar immunogenicity with natural viral particles. Thus, VP2 was selected for the in vivo experiment.

The host innate immune response is the primary mechanism for resisting and clearing viruses during the early stage of infection (Barjesteh et al., 2015). The acquired immunity in vaccinated animals likely alters the typical innate im- mune response profile (Pereiro et al., 2014). Many papers have been published on GPV humoral immune responses, and the selection of VP proteins as antigen targets for a GPV vaccine has been previously established. However, little is known about cellular immunity against GPV. The pcDNA-GPV-VP1 DNA vaccine was shown to induce both cellular and humoral responses, and it can induce higher cellular and humoral immunity than live attenuated vaccine (Deng et al., 2014). The codon-optimized VP2 (optVP2) is capable of forming VLPs, which are highly immunogenic. Immunogenicity assays revealed that VLPs with optVP2 could induce a high level of antibody production and provide effective protections against lethal challenge (Chen et al., 2012). Moreover, Lee et al. (2010) selected some different adjuvants formulated with VP2 from GPV. Those results suggested that the percentage of $\mathrm{CD}^{+} / \mathrm{CD}^{+}$cells in the PBMCs was significantly increased in ducks immunized with VP2 that was formulated with CpG ODNs. The major capsid protein VP3 was also tested in animals. Wang et al. (2015) constructed a recombinant VP3 vaccine (rmNA-VP3) by using the avirulent strain LaSota as a vaccine vector. Goslings inoculated with rmNA-VP3 showed no apparent signs of disease and had high levels of anti-GPV and antiNDV neutralizing antibodies. Additionally, the localization of B-cell epitopes on GPV capsid proteins has been defined (Yu et al., 2012). In other parvoviruses, for example human bocavirus species, the immunodominant epitopes of VP2 protein were also determined. Two of the peptides contain conserved epitopes among human bocavirus 1-4; these were recognized by a human polyclonal antibody and elicited high titer of antibodies in mice (Zhuo et al., 2014).

Based on the immunobiological activity of VPs in vitro, humoral immune responses induced by GPV-VP2 were further explored in vivo. Here, we measured transcripts of some immune-related genes, such as $\mathrm{T}$ lymphocytes markers (CD4 and CD8 $\alpha$ ), IL-1 $\beta$, IL-6, and IFNs after vaccination in goslings. $\mathrm{T}$ cell-mediated immunity is the central element of the adaptive immune system. Virus-specific $\mathrm{CD} 8 \mathrm{a}^{+} \mathrm{T}$ cells have been shown to be important for the elimination of viral shedding (Reusser et al., 1991; McNeal et al., 1995). In our study, the CD4 and CD8a mRNA expression levels increased over time after immunization. The expression of CD8 a significantly increased on day 7 and day 14 , suggesting that our VP2 vaccine induced a strong immune response at the early stage. Pro-inflammatory cytokines such as IL- $1 \beta$ play an important role in host immune and inflammatory reactions. In this study, we found that the mRNA levels of CD8a, IL-1 $\beta$, IFN $\gamma$, and IFN $\lambda$ in goose PBMCs were highly increased in the early phase of vaccination with pcDNA3.1(+)-VP2, which could compensate for the low humoral immunity (low titers of GPV-specific antibody) in the early post-vaccination phase. The antigen-specific humoral responses, particularly against VP2 and VP3, are 
crucial for the prevention of viral assembly. The enhanced $\mathrm{T}$ cell population should promote stronger humoral responses. These data suggested that vaccination with pcDNA3.1(+)VP2 had a strong cellular immune reactivity and induced strong humoral responses. These data will serve as a basis for better understanding of the molecular processes needed for successful vaccination development.

Acknowledgments. This work was funded by grants from the Innovative Research Team Program in Education Department of Sichuan Province (No. 2013TD0015), the National Science and Technology Support Program (No. 2015BAD12B05), the Integration and Demonstration of Key Technologies for the Duck Industry in Sichuan Province (No. 2014NZ0030), and China Agricultural Research System (CARS-43-8).

\section{Reference}

Barjesteh N, Shojadoost B, Brisbin JT, Emam M, Hodgins DC, Nagy É, Sharif, S (2015): Reduction of avian influenza virus shedding by administration of toll-like receptor ligands to chickens. Vaccine 33, 4843-4849. https://doi. org/10.1016/j.vaccine.2015.07.070

Chen S, Zhao Q, Qi Y, Liu F, Wang M, Jia R, Zhu D, Liu M, Chen X, Cheng, A (2015): Immunobiological activity and antiviral regulation efforts of chinese goose (anser cygnoides) $c d 8 \alpha$ during ngvev and gpv infection. Poult. Sci. 94, 17-24. https://doi.org/10.3382/ps/peu024

Chen Z, Li C, Zhu Y, Wang B, Meng C, Liu G (2012): Immunogenicity of virus-like particles containing modified goose parvovirus VP2 protein. Virus Res. 169, 306-309. https:// doi.org/10.1016/j.virusres.2012.08.009

Chipman P R, Agbandje-Mckenna M, Kajigaya S, Brown KE, Young NS, Baker TS, Rossmann MG (1996): Cryo-electron microscopy studies of empty capsides of human parvovirus b19 complexed with its cellular receptor. Proc. Natl. Acad. Sci. USA 93, 7502-7506. https://doi.org/10.1073/ pnas.93.15.7502

Cotmore SF, Agbandjemckenna M, Chiorini JA, Mukha DV, Pintel DJ, Qiu J, Soderlundvenermo M, Tattersall P, Tijssen P, Gatherer D (2014): The family parvoviridae. Arch. Virol. 159, 1239-1247. https://doi.org/10.1007/s00705013-1914-1

Deng SX, Cai MS, Cui W, Huang JL, Li ML (2014): Evaluation of the immune response in shitou geese (anser anser domesticus) following immunization with gpv-vp1 dna-based and live attenuated vaccines. Vet. Q. 34, 180-184. https:// doi.org/10.1080/01652176.2014.966173

Gall-Reculé, G. L., \& Jestin, V (1994): Biochemical and genomic characterization of muscovy duck parvovirus. Arch. Virol. 139, 121-131. https://doi.org/10.1007/BF01309459

Lee J, Lin YM, Yen T, Yang WJ, Chu CY (2010): CpG oligodeoxynucleotides containing gacgtt motifs enhance the immune responses elicited by a goose parvovirus vaccine in ducks. Vaccine 28, 7956-7962. https://doi.org/10.1016/j.vaccine.2010.09.072
Jansson DS, Feinstein R, Kardi V, Mató T, Palya V (2007): Epidemiologic investigation of an outbreak of goose parvovirus infection in Sweden. Avian Dis. 51, 609-613. https://doi. org/10.1637/0005-2086(2007)51[609:EIOAOO]2.0.CO;2

Jin H, Xia X, Liu B, Fu Y, Chen X, Wang H, Xia Z (2012): Highyield production of canine parvovirus virus-like particles in a baculovirus expression system. Anal. Biochem. 394, 110-116.

Ju H, Wei N, Wang Q, Wang C, Jing Z, Guo L, Liu D, Gao M, Ma B, Wang J (2011): Goose parvovirus structural proteins expressed by recombinant baculoviruses self-assemble into virus-like particles with strong immunogenicity in goose. Biochem. Biophys. Res. Commun. 409, 131-136. https://doi.org/10.1016/j.bbrc.2011.04.129

Lombardo E, Ramírez JC, Garcia J, Almendral JM (2002): Complementary roles of multiple nuclear targeting signals in the capsid proteins of the parvovirus minute virus of mice during assembly and onset of infection. J. Virol. 76, 7049. https://doi.org/10.1128/JVI.76.14.7049-7059.2002

Lowin T, Raab U, Schroeder J, Franssila R., Modrow S (2010): Parvovirus B19 VP2-proteins produced in Saccharomyces cerevisiae: comparison with VP2-particles produced by baculovirus-derived vectors. Zoonoses Public Health 52, 348-352.

Mcneal MM, Barone KS, Rae MN, Ward RL (1995): Effector functions of antibody and cd $8+$ cells in resolution of rotavirus infection and protection against reinfection in mice. Virology 214, 387. https://doi.org/10.1006/ viro.1995.0048

Palya V, Zolnai A, Benyeda Z, Kovács E, Kardi V, Mató T (2009): Short beak and dwarfism syndrome of mule duck is caused by a distinct lineage of goose parvovirus. Avian Pathol. 38, 175. https://doi.org/10.1080/03079450902737839

Pereiro P, Dios S, Bolta-a S, Coll J, Estepa A, Mackenzie S, Novoa B, Figueras A (2014): Transcriptome profiles associated to vhsv infection or dna vaccination in turbot (scophthalmus maximus). Plos One 9, e104509. https://doi.org/10.1371/ journal.pone.0104509

Qi Y, Chen S, Zhao Q, Wang M, Jia R, Zhu D, Liu M, Liu F, Chen X, Cheng A (2015): Molecular cloning, tissue distribution, and immune function of goose tlr7. Immunol. Lett. 163, 135. https://doi.org/10.1016/j.imlet.2014.11.017

Reusser P, Riddell SR, Meyers JD, Greenberg PD (1991): Cytotoxic t-lymphocyte response to cytomegalovirus after human allogeneic bone marrow transplantation: pattern of recovery and correlation with cytomegalovirus infection and disease. Blood 78, 1373.

Ros C, Gerber M, Kempf C (2006): Conformational changes in the vp1-unique region of native human parvovirus b19 lead to exposure of internal sequences that play a role in virus neutralization and infectivity. J. Virol. 80, 12017. https:// doi.org/10.1128/JVI.01435-06

Summerford C, Samulski RJ (1998): Membrane-associated heparan sulfate proteoglycan is a receptor for adeno-associated virus type 2 virions. J. Virol. 72, 1438.

Vihinenranta M, Wang D, Weichert WS, Parrish CR (2002): The vp1 $\mathrm{n}$-terminal sequence of canine parvovirus affects nuclear transport of capsids and efficient cell infection. J. Virol. 
76, 1884-1891. https://doi.org/10.1128/JVI.76.4.1884$\underline{1891.2002}$

Wang J, Cong Y, Yin R, Feng N, Yang S, Xia X et al. (2015): Generation and evaluation of a recombinant genotype vii newcastle disease virus expressing vp3 protein of goose parvovirus as a bivalent vaccine in goslings. Virus Res. 203, 77-83. https://doi.org/10.1016/j.virusres.2015.04.006

Xu J, Guo HC, Wei YQ, Dong H, Han SC, Ao D, Sun DH, Wang HM, Cao SZ, Sun SQ (2014): Self-assembly of virus-like particles of canine parvovirus capsid protein expressed from escherichia coli, and application as virus-like particle vaccine. Appl. Microbiol. Biotechnol. 98, 3529. https:// doi.org/10.1007/s00253-013-5485-6
Yu TF, Ma B, Gao MC, Wang JW (2012): Localization of linear b-cell epitopes on goose parvovirus structural protein. Vet. Immunol. Immunopathol. 145, 522-526. https://doi. org/10.1016/j.vetimm.2011.11.022

Zhou H, Yao G, Cui S (2010): Production and purification of VP2 protein of porcine parvovirus expressed in an insectbaculovirus cell system. Virology J. 7, 366. https://doi. org/10.1186/1743-422X-7-366

Zhuo Z, Xin G, Wang Y, Zhou H, Chao W, Paranhosbaccalà G, Vernet G, Li G, Wang J (2014): Conserved b-cell epitopes among human bocavirus species indicate potential diagnostic targets. Plos One 9, e86960. https://doi. org/10.1371/journal.pone.0086960 